\title{
A temporally dynamic context effect that disrupts voice onset time discrimination of rapidly successive stimuli
}

\author{
JACQUELINE LIEDERMAN \\ Boston University, Boston, Massachusetts \\ RICHARD FRYE \\ Children's Hospital, Boston, Massachusetts \\ and \\ JANET MCGRAW FISHER, KIMBERLY GREENWOOD, and REBECCA ALEXANDER \\ Boston University, Boston, Massachusetts
}

\begin{abstract}
Across three experiments, voice onset time discrimination along a $/ \mathrm{ba} /-/ \mathrm{pa} /$ continuum was found to be influenced by the order of presentation of rapidly successive stimuli. Specifically, discrimination was disrupted when a relatively unambiguous /pa/ syllable was presented before, rather than after, a more ambiguous /pa/ or /ba/syllable. In Experiments 1 and 2, for between-category discrimination, this order effect was significant at interstimulus intervals (ISIs) below $250 \mathrm{msec}$, but not at 250 or 1,000 msec. In Experiments 2 and 3, the order effect was also significant for within-category discrimination at ISIs below 250 msec. In addition, in Experiment 3 this order effect was not diminished by provision of performance feedback across eight testing sessions. These findings reveal a particular vulnerability of phonological processing in response to rapidly successive stimuli and may have implications for mathematical and neural models of speech processing of normal and impaired populations.
\end{abstract}

A variety of context effects have been reported in the speech perception literature, such as adaptation effects (Eimas \& Corbit, 1973), lexical effects (Allen \& Miller, 2001), contrast effects (Diehl, Elman, \& McCusker, 1978), and order effects (Lotto, Kluender, \& Holt, 1998). Perhaps the best known is the perceptual magnet effect (Iverson \& Kuhl, 1996; Kuhl, 1991, 1994), whose interpretation remains controversial (e.g., Lotto, Kluender, \& Holt, 1998). Some describe it as a distortion of perceptual space, such that the best exemplars of a phonemic category magnetically attract neighboring exemplars. This results in poorer discrimination of acoustic differences near the best exemplars of a category and better discrimination of differences near the poor exemplars. Indeed, Iverson and Kuhl (1996) demonstrated that near the best category exemplars, $d^{\prime}$ is minimal and perceptual clustering is greatest, whereas near boundaries between categories, where exemplars are more ambiguous, $d^{\prime}$ is large and perceptual clustering is minimal.

Originally, Kuhl (1991) used an adaptation paradigm in which a single vowel was presented repeatedly, followed by a second vowel differing slightly in first and second formant frequencies. Differences between the vowels were harder to perceive when the initial vowel

Correspondence should be addressed to J. Liederman, Brain, Behavior and Cognition Program, Boston University, 64 Cummington St., Boston, MA 02215 (e-mail: liederma@bu.edu). was a good category exemplar, rather than a poor one. Our goal was to examine the temporal properties of this context effect in neurologically intact adults so as to eventually evaluate these parameters within individuals with various kinds of phonological processing impairments. We changed the stimuli from vowels to consonantvowel (CV) syllables that varied in voice onset time (VOT). Our decision to use CV syllables was fueled by claims that disrupted discriminative abilities for VOT continua are found in individuals with language learning deficits (Tallal \& Piercy, 1974; Thibodeau \& Sussman, 1979) and/or dyslexia (Adlard \& Hazan, 1998; Breier et al., 2001; Manis et al., 1997; Masterson, Hazan, \& Wijayatilake, 1995; Mody, Studdert-Kennedy, \& Brady, 1997; Werker \& Tees, 1987). We also modified the original paradigm by using only two tokens in each trial.

The critical variation in our paradigm was to examine the temporally dynamic properties of the effect of order of presentation of the two stimuli to be discriminated. Macmillan, Goldberg, and Braida (1988) have argued that some stimuli along a dimension play a special role in providing a reference point that is used in the contextual coding of other stimuli. Both category boundaries (e.g., Diehl, Lang, \& Parker, 1980) and prototypes (Samuel, 1982) have been suggested as critical. We chose distance from the categorical $/ \mathrm{ba} /-/ \mathrm{pa} /$ boundary as such a reference point, since Samuel demonstrated that for VOT stimuli like ours, distance from the boundary accounted for se- 
lective adaptation effects. As will be seen, categorization and performance data from our three experiments validate our classification of tokens as relatively unambiguous or ambiguous exemplars of the category /pa/. Thus, we examined the context effect of whether an unambiguous exemplar of the /pa/ category was presented first or second.

Also important was our decision to vary the duration of the interval between the two successive syllables. This decision was motivated by reports that subgroups of the aforementioned populations have special difficulty processing brief stimuli in rapid temporal succession (for a review, see Habib, 2000).

\section{EXPERIMENT 1}

We sought to develop a VOT continuum that contained at least two syllable categories, with at least one category containing enough tokens for within-category discrimination to be studied. After the appropriate VOT continuum was constructed, categorization and discrimination of the tokens was examined. The effect of the quality of the first token on perception of the second token was studied as a function of ISI.

\section{Method}

\section{Participants}

The participants were 51 native English-speaking college students (20 males, 31 females) with no known history of reading or neurological abnormalities and a mean age of 19 years.

\section{Stimuli}

An 11-token /ba/ to /pa/ VOT continuum was constructed from stimuli on the University of Nebraska Speech Perception Laboratory Resources Web site (http://hush.unl.edu/LabResources.html).
The first token had a VOT of $0 \mathrm{msec}$, meaning that the onset of the release burst and the onset of voicing was simultaneous. Each successive token represented about a 5-msec increase in VOT. See the Appendix for a more complete description of the stimuli.

\section{VOT Categorization Task}

The participants heard each of the 11 tokens 10 times, in a randomized order, and categorized them as /ba/ or /pa/ by pressing one of two keys. Half were presented at the beginning of the session and half at the end of the session.

\section{VOT Discrimination Task}

The VOT discrimination task was designed with a roving standard in a match versus mismatch format. The participants pressed one of two keys to indicate whether the two sequentially presented stimuli were identical or not. Forty trials were presented at each of six ISIs: $10,25,100,250,1,000$, and 2,500 msec. These ISIs were selected to span the period between backward masking (i.e., at short ISIs, such as 10 and $25 \mathrm{msec}$ ) and phonological working memory (i.e., at longer ISIs, such as 1,000 and 2,500 msec). Stimuli matched on $50 \%$ of the trials, whereas stimuli differed by three to eight tokens during the other $50 \%$ of the trials, depending upon the participant's discrimination performance. The difference necessary to maintain each participant's overall performance above $70 \%$ correct using an ISI of $250 \mathrm{msec}$ was determined using a staircase method during the initial 50-100 practice trials. The succeeding 240 trials were distributed between the six ISIs at the VOT difference chosen. All token combinations that produced pairs at the designated VOT difference were presented.

\section{Results}

\section{Categorization}

The categorization data from the beginning and end of the session were averaged. Figure 1 indicates that virtually $100 \%$ of the time, Tokens $1-3$ were consistently categorized as /ba/, whereas Tokens 6-11 were consistently categorized as $/ \mathrm{pa} /$. Tokens 4 and 5 were considered

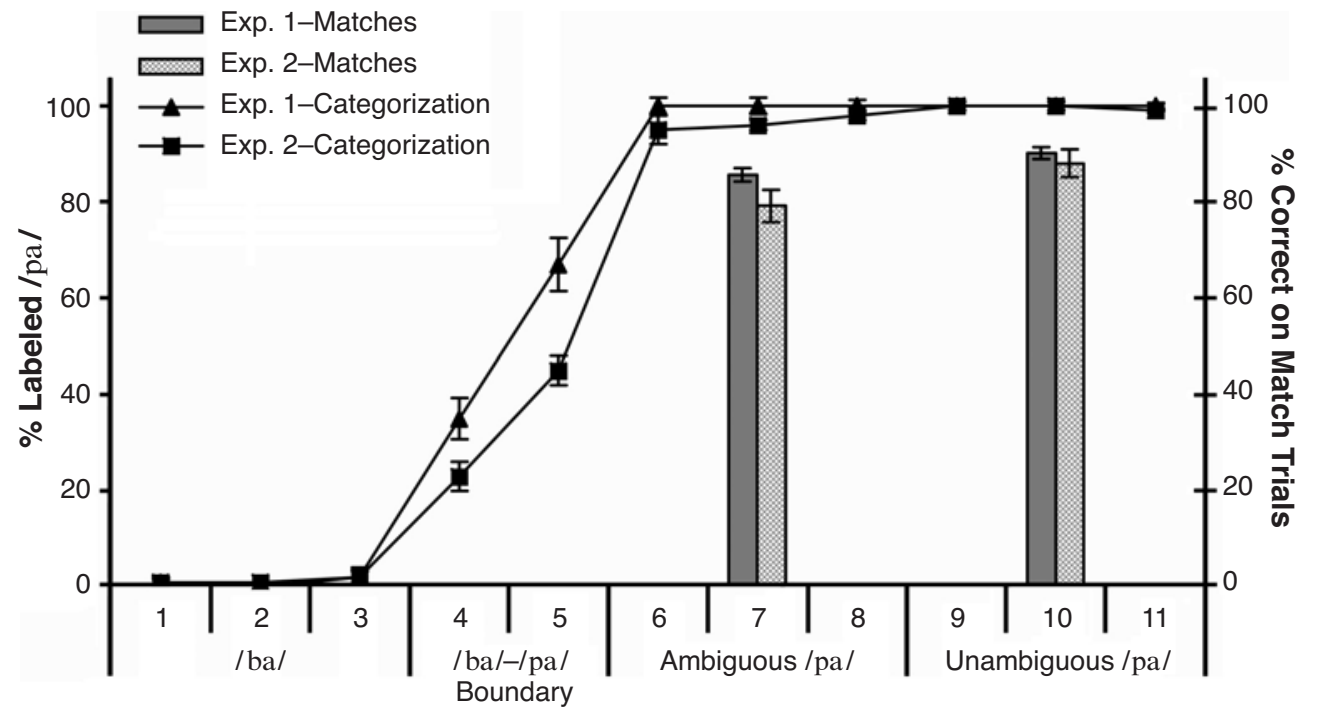

Figure 1. Categorization, indicated by the line graph for Experiments 1 and 2: Tokens 1-3 are labeled as /ba/, Tokens 6-11 are labeled as /pa/, and Tokens 4 and 5 are at the /ba/-/pa/ boundary. Discrimination, indicated by the bar graph for Experiments 1 and 2 for matching trials: Responses to ambiguous /pa/ tokens proximal to the boundary $(6,7,8)$ are significantly less accurate than are responses to the relatively unambiguous $/ \mathrm{pa} /$ tokens distal to the boundary $(9,10,11)$. 
boundary tokens since they were inconsistently categorized as either $/ \mathrm{ba} / \mathrm{or} / \mathrm{pa} /$.

The VOT region for the /pa/ tokens was subdivided on the basis of distance from the boundary in order to study within-category discrimination. Thus, Tokens $6-8$ were considered proximal to the $/ \mathrm{ba} /-/ \mathrm{pa} /$ category boundary, whereas Tokens $9-11$ were distal to the /ba/-/pa/ boundary.

Thus, four VOT regions were created: the proximal versus distal /pa/ tokens, the tokens labeled /ba/, and the boundary items, which were labeled /ba/ or $/ \mathrm{pa} /$.

\section{Discrimination}

Data reduction. Few participants received token pairs derived solely from the six token /pa/ portions of the continuum. Therefore, analysis of the mismatch trials was restricted to between-category trials - that is, those trials for which (1) the distal /pa/ token $(9,10$, or 11) occurred either in the first or second position, and (2) the comparison token was not consistently labeled as /pa/ (i.e., /ba/ Tokens 1, 2, or 3 or boundary Tokens 4 or 5).

Data from the extremely long 2,500-msec ISI trials were dropped from the analyses if the participant responded before the onset of the second stimulus. Since few data points existed once anticipatory trials were omitted, all of the ISI 2,500-msec data were excluded from further analysis.

Match trials. Figure 1 demonstrates that accuracy for distal $/ \mathrm{pa} /$ match trials was significantly better $(M=$ $90.2, S E=.013)$ than for proximal $/ \mathrm{pa} /$ match trials $[M=85.6, S E=.014 ; F(1,50)=15.34, p<.001]$. Two identical tokens were misjudged as different more often when they derived from the proximal rather than the distal VOT region. In other words, Tokens 9-11 were more accurately matched to themselves than were Tokens 6-8.

Mismatch trials. On average, participants required stimuli to be five tokens apart in order to achieve an overall performance of $70 \%$ correct. This difference was too large to permit an adequate number of within-category mismatch discrimination trials for analysis.

As can be seen in Figure 2A, a within-subjects analysis of variance (ANOVA) for the between-category trials demonstrated that accuracy was affected by ISI $[F(1,50)=$ $33.578, p=.000]$ and order of presentation of the distal $/ \mathrm{pa} /$ token [i.e., first or second in the pair; $F(4,200)=$ $9,306, p<.001]$. These main effects, however, were modified by an order $\times$ ISI interaction $[F(4,200)=$ $7.325, p=.000]$. Only for the three shortest ISIs was performance significantly lower $(p<.01$, after Bonferroni correction) when the distal $/ \mathrm{pa} /$ was in the first rather than the second position. Indeed, the difference in accuracy between Orders 1 and 2 was $30.8 \%$ for ISI $10 \mathrm{msec}$, $31.2 \%$ for ISI $25 \mathrm{msec}$, and $21.1 \%$ for ISI $100 \mathrm{msec}$. In contrast, the order effects at the longer ISIs of 250 and $1,000 \mathrm{msec}$ were less than $1 \%$.

\section{Discussion}

Experiment 1 confirmed that the tokens within the $/ \mathrm{pa} /$ category could be divided into perceptually distinct regions. Matching accuracy was better for identical pairs of distal / $\mathrm{pa}$ / than for proximal /pa/tokens. As a way to refer to these two distinct VOT regions, the distal /pa/ tokens hereafter are referred to as relatively unambiguous and the proximal /pa/ tokens as relatively ambiguous.

When a relatively unambiguous /pa/ token occurred first, rather than second, in discrimination trials of betweencategory tokens, performance was reduced by as much as $30 \%$ (Figure 2A). Moreover, this order effect occurred only within a specific temporal window: It was robust at ISIs of 10,25 , and $100 \mathrm{msec}$, but the effect was not significant at 250 and $1,000 \mathrm{msec}$.

\section{EXPERIMENT 2}

Experiment 2 was designed to provide a sufficient number of trials for the reevaluation of the order effect for both within- and between-category discrimination. This was accomplished by using a fixed protocol with pairs of mismatch tokens that were 4 to 7 tokens apart. We also included an unambiguous /pa/ in the first or second position of every mismatch trial.

\section{Method}

\section{Participants}

The selection criteria were the same as those used in Experiment 1 . There were 18 participants ( 6 males, 12 females), with a mean age of 20.8 years.

\section{Stimuli}

The stimuli were the same as those used in Experiment 1.

\section{Procedure}

The categorization and discrimination procedures were the same as in Experiment 1, except for the following modifications. Mismatch trials were restricted to VOT differences of 4, 5, 6, or 7 tokens for the between-category trials and 4 or 5 tokens for the within-category trials. ISIs of 25,250 , and $1,000 \mathrm{msec}$ were used. There were a total of 540 trials, half match and half mismatch.

\section{Results}

\section{Categorization}

The categorization results from Experiment 2 replicate those from Experiment 1, further justifying the division of the VOT continuum into the four distinct subregions of /ba/, boundary, ambiguous /pa/, and unambiguous /pa/ (see Figure 1).

\section{Discrimination}

Match trials. Consistent with the results from Experiment 1, Figure 1 shows that percentage correct for match $/ \mathrm{pa} /$ trials was significantly better for identical pairs of relatively unambiguous /pa/ tokens $(M=88.6, S E=$ $.029)$ than for pairs of ambiguous /pa/ tokens $[M=79.9$, $S E=.029 ; F(1,17)=19.356, p<.001]$.

Mismatch trials. A within-subjects ANOVA with order (relatively unambiguous token in first vs. second position) and ISI as factors indicated that for the betweencategory trials, there was a main effect of order $[F(1,17)=$ 9.496, $p=.007]$, which was modified by an interaction with ISI $[F(2,34)=9.180, p=.001]$ on the percentage correct. As can be seen in Figure 2B, the order effect was 

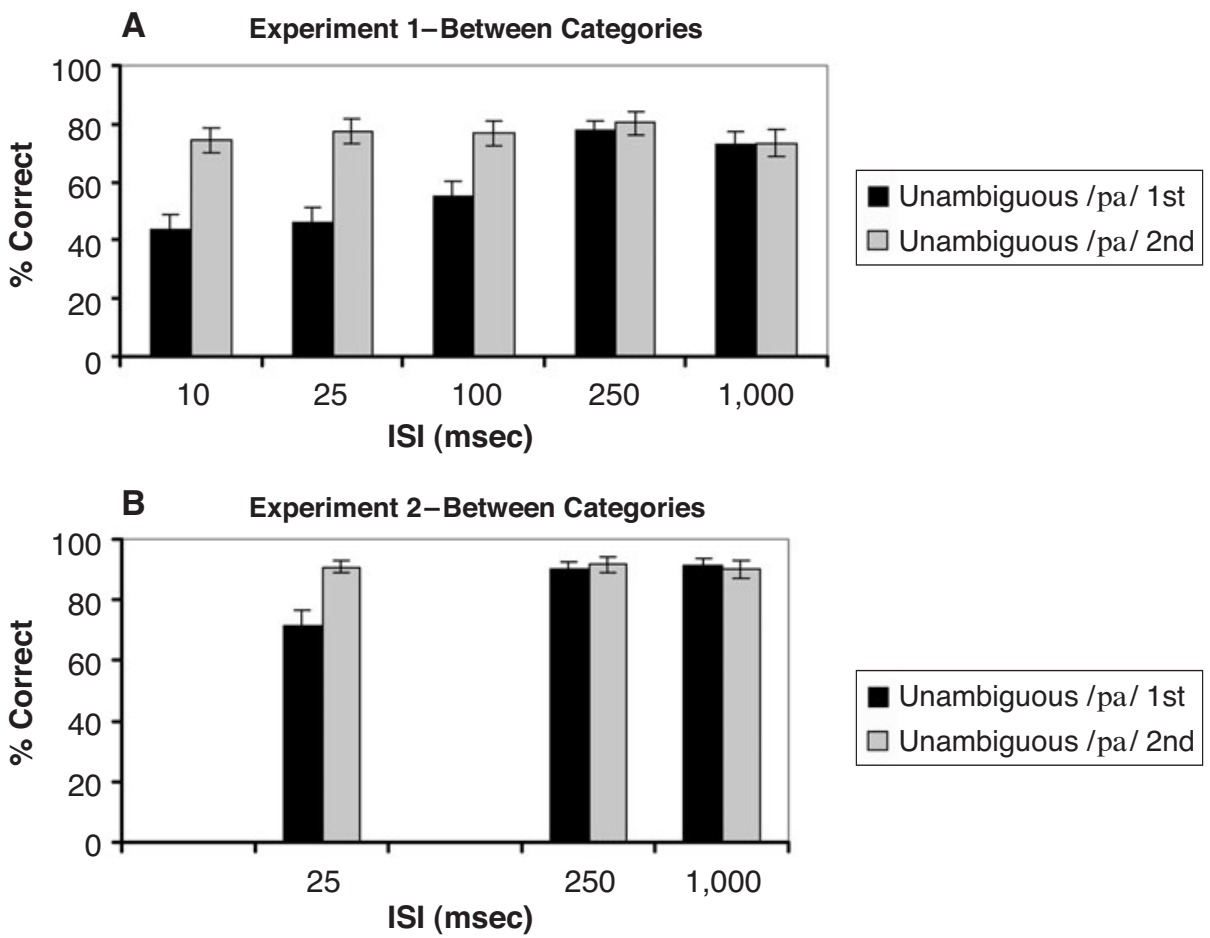

Unambiguous /pa/1st

Unambiguous /pa/ 2nd

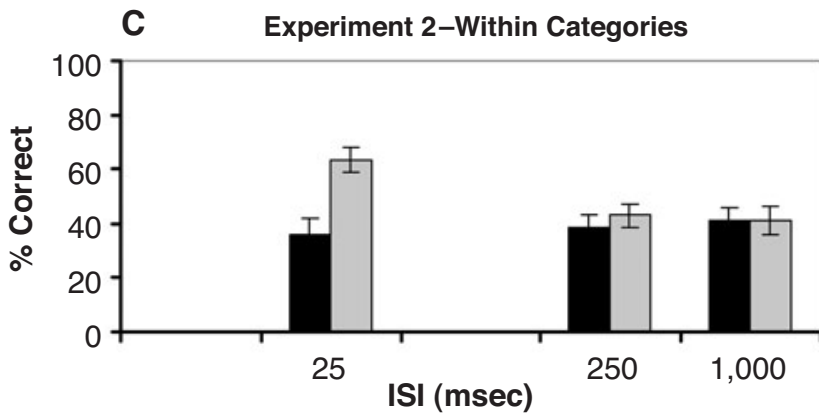

Unambiguous /pa/1st

Unambiguous /pa/2nd

Figure 2. Experiments 1 and 2, discrimination during mismatch trials: Accuracy is greater when the relatively unambiguous item is in the second rather than the first position. In both experiments, this order effect is significant for ISIs below $250 \mathrm{msec}$ only.

significant at ISI $25 \mathrm{msec}[M($ Order 1$)=71.3, S E=5.7$; $M($ Order 2$)=90.9, S E=2.5]$, but not at ISIs of 250 and $1,000 \mathrm{msec}$.

As can be seen in Figure 2C, for the within-category trials, an order $\times$ ISI interaction was observed $[F(2,34)=$ $10.923, p<.001]$ on percentage correct. Again, the order effect was significant at ISI $25 \mathrm{msec}[M($ Order 1$)=36$; $S E=6.4 ; M($ Order 2$)=63.6, S E=4.5]$, but not at ISIs of 250 and $1,000 \mathrm{msec}$.

\section{Discussion}

Experiment 2 replicated the results from Experiment 1 and demonstrated that the order effect was modulated by ISI for both within-category and between-category discrimination. Further examination of the temporal dynamics of these effects will be deferred to the General Discussion section.

\section{EXPERIMENT 3}

The order effect we have demonstrated in the two previous experiments appears to be a robust and replicable finding. The next question was whether this order effect could be unlearned or modified by feedback about performance or by providing multiple sessions of experience. This experiment was confined to the shortest ISIs, where the effect was found to be strongest.

\section{Method}

\section{Participants}

The selection criteria were the same as those used in Experiments 1 and 2. There were 6 participants ( 3 males, 3 females), with a mean age of 20.6 years.

\section{Stimuli}

The stimuli were the same as those used in Experiments 1 and 2. 


\section{Procedure}

The stimuli were presented across eight 486 -trial sessions. Feedback alternated between sessions, with half of the participants starting with feedback and half starting without feedback. VOT differences of $2,3,4$, and 5 tokens were used for mismatch trials. ISIs of 10 , 20 , and $30 \mathrm{msec}$ were selected to further examine the temporal window within which the strongest order effects were observed in Experiments 1 and 2 .

Between-category trials consisted of those token pairs that contrasted an unambiguous /pa/ with a non/pa/ (i.e., a /ba/ or boundary token). These trials began or ended with an unambiguous $/ \mathrm{pa} /$. Within-category trials contained those pairs that contrasted two $/ \mathrm{pa} /$ tokens, beginning or ending with an unambiguous /pa/ token $(9,10$, or 11$)$ and having an ambiguous /pa/ token $(6,7$, or 8$)$ in the other position.

\section{Results}

\section{Data Reduction}

No significant main effects or interactions with feedback were found. Feedback was dropped as a factor, and successive sessions were combined to create four sessions of 992 trials each.

\section{Discrimination}

Match trials. Once again, accuracy of matching pairs of relatively unambiguous tokens $(M=90, S E=3.80)$ was $18 \%$ greater than for matching pairs of ambiguous $/ \mathrm{pa} /$ tokens $[M=72, S E=3.35 ; F(1,5)=19.60, p=.007]$.

Mismatch trials. Across all of these brief ISIs, accuracy was lower when a relatively unambiguous token was presented first $(M=51.7, S E=.045)$ rather than second $[M=75.9, S E=.028 ; F(1,5)=237.50, p<.001$; see Figure 3]. To further examine the effect of session and ISI, the magnitude of the order effect was calculated as the difference in percentage correct during Orders 2 ver- sus 1 divided by the sum of the percentage correct during Orders 2 and 1. Within- and between-category trials were analyzed separately.

For the between-category trials, the magnitude of the order effect did not significantly change with ISI or session. For the within-category trials, the magnitude of the order effect increased as ISI increased from 10 to $30 \mathrm{msec}$ $[F(1,5)=38.343, p=.002]$ and increased with successive sessions $[F(1,5)=11.83, p=.02$; Figure 3, left and right panels, respectively].

\section{GENERAL DISCUSSION}

A context effect is described such that VOT discrimination is significantly attenuated when a relatively unambiguous token is presented first, rather than second, within a pair of CV syllables. This context effect was replicated in all three experiments, for both within-category and between-category discriminations. Thus, the order of presentation of the relatively unambiguous /pa/ token was critical, not whether the other token was another/pa/ or $/ \mathrm{ba} /$.

Of special interest were the temporal dynamics of this order effect. These dynamics were quite orderly and reliable. The order effect was observed and replicated for between-category trials only at ISIs $<250 \mathrm{msec}$. A similar order effect was observed for within-category trials only at ISIs $<250 \mathrm{msec}$. The dynamics of this effect at very short ISIs were demonstrated in Experiment 3. This order effect may not be well known, since the typical ISI in VOT discrimination studies is $250 \mathrm{msec}$.

The order effect could not be unlearned within the constraints of Experiment 3. Trial-by-trial feedback did
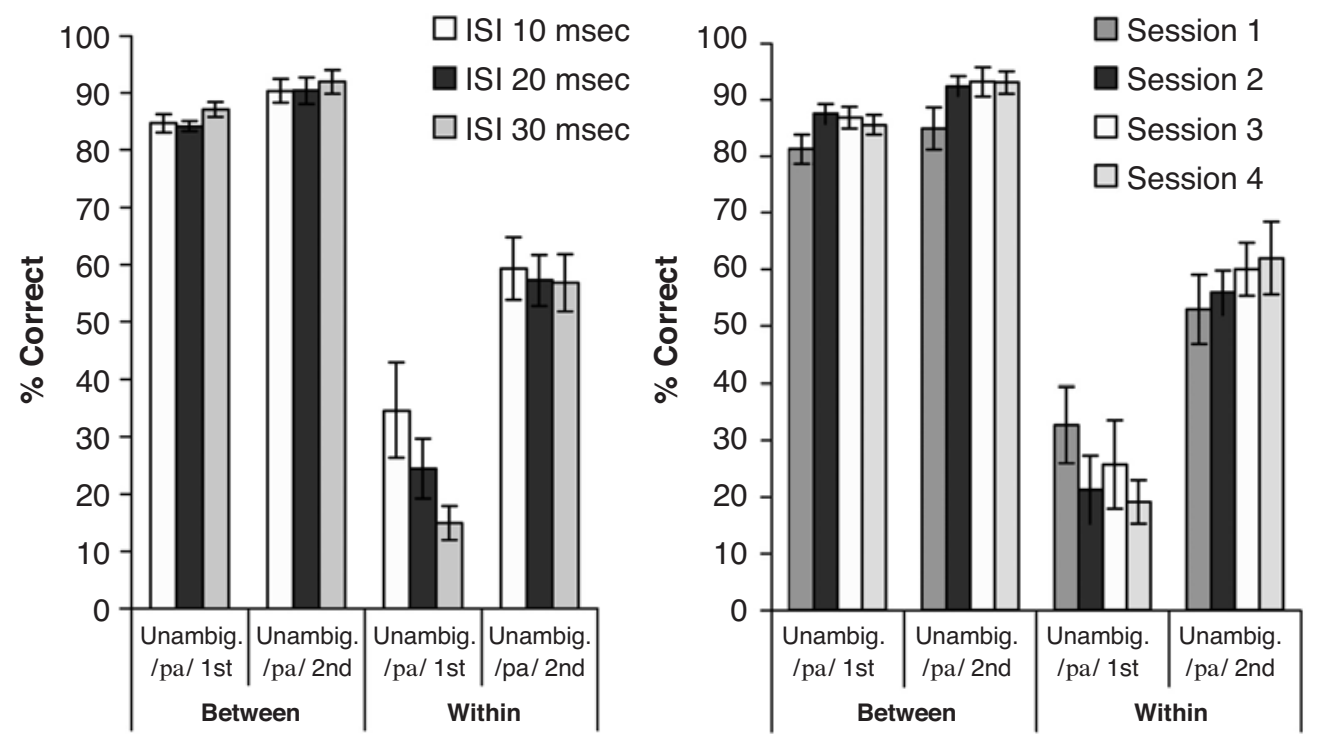

Figure 3. Experiment 3, discrimination data during mismatch trials: Accuracy is higher when the relatively unambiguous item is in the second rather than the first position in a pair, for both between- and within-category trials. This order effect increases with ISIs from 10 to $30 \mathrm{msec}$ (left) and session (right) for the within-category trials only. 
not alter the order effect. Moreover, experience extended over four sessions served to significantly strengthen, rather than diminish, this effect for the within-category trials. This indicates that the effect was not simply due to unfamiliarity with these stimuli; on the contrary, familiarity strengthened the bias.

Several features of the present experiments enabled us to establish and characterize this order effect. First, the context of the trial varied due to the use of a roving, rather than a fixed, standard. Second, the VOT continuum contained six tokens that were uniformly categorized as /pa/. Relatively unambiguous and ambiguous /pa/ tokens were defined and verified by their differential matching accuracy. Finally, by moving away from adaptation paradigms, the ISI could be varied over a broad range $(10-1,000 \mathrm{msec})$.

None of the previously reported contextual effects (Allen \& Miller, 2001; Diehl, Elman, \& McCusker, 1978; Eimas \& Corbit, 1973; Iverson \& Kuhl, 2000; Lotto \& Kluender, 1998) have been examined in terms of their temporal dynamics. Indeed, only some mathematical models of speech perception have tried to address changes in performance due to changes in ISI (e.g., Macmillan, Goldberg, \& Braida, 1988; Schouten \& van Hessen, 1992). As will be described below, we suggest that a neural network model of adaptive resonance can account for the dynamic properties of the order effect and stored category representations along the lines of the hierarchical bidirectional model proposed by Grossberg and Myers (2000).

Such a model would have to accommodate to the notion that two different temporal processes can account for the influence of one token upon its discrimination from a second token. The first process is the time required for an adaptively resonant neural network to change from its baseline to a stabilized state in response to a signal. As will be argued, the data suggest that this time is inversely proportional to the ambiguity of the token. Since the order effect is seen within $10 \mathrm{msec}$, it is reasonable to propose that the network begins to stabilize in response to a relatively unambiguous token within $10 \mathrm{msec}$. The second process is the time required for the network to label the signal. We suggest that labeling of the signal has occurred by $250 \mathrm{msec}$. Thus, performance across all three experiments can be accounted for by a combination of four parameters: (1) the initial state of the network, which, according to Grossberg and Myers (2000), is nonresonant; (2) the degree of ambiguity of the initial /pa/ token; (3) whether the second token, if presented in isolation, would elicit a /pa/ label or not; and (4) the ISI between the first and second tokens.

For example, the order effect did not occur when the ISI was $\geq 250 \mathrm{msec}$. We suggest that $250 \mathrm{msec}$ is enough time for the token to adequately stabilize the network, be associated with a category label, and permit the network to reset back to its resting state prior to receipt of the second token. Under such circumstances, it does not matter which token is presented first.

Indeed, it is worth noting that for ISIs $\geq 250 \mathrm{msec}$, irrespective of order, between-category discrimination remains quite accurate but within-category discrimination deteriorates to below-chance levels. We suggest that the process of labeling facilitates between-category discrimination but actually interferes with within-category discrimination. Thus, for long ISIs between-category discrimination remains accurate. However, successive within-category tokens are erroneously judged to be matching, since the two tokens are both labeled as /pa/.

In contrast, when ISIs were $<250 \mathrm{msec}$, the order effect was prominent for both between- and within-category discriminations. These short ISIs may provide enough time for the network to stabilize in response to a relatively unambiguous token, but not enough time for labeling and resetting to occur. For example, a relatively unambiguous /pa/ may quickly stabilize the network. Therefore, the second token must influence a network that is in an initially stable state, rather than a resting state. If the subsequent token is a within-category ambiguous /pa/, it may have little ability to destabilize a network that has already been stabilized. This lack of change of state in the network may induce a strong bias against detecting that the two successive stimuli differed.

However, if a relatively ambiguous token is presented first, the network may not have sufficient time to stabilize before the second unambiguous token enters the network. Since the second unambiguous token quickly stabilizes the network, a significant change in the network state is detected and the items are correctly judged as different. Thus, within-category discrimination is actually above chance levels at the shortest ISIs, as long as the ambiguous token is presented first.

One other temporal parameter was observed for the shortest ISIs tested in Experiment 3. Between 10 and $30 \mathrm{msec}$, the order effect was robust and stable for the between-category trials but increased in magnitude for the within-category trials. This suggests that the network is continuing to stabilize in response to a relatively unambiguous / $\mathrm{pa} /$ signal within this time frame. If the second item is another /pa/, the probability of change detection decreases. However, within this time interval, if the second item is not a $/ \mathrm{pa} /$, the probability of change detection is great enough that it is unaffected by ISI.

The results of these experiments indicate that assessments of phonological processing ability must take into account the rate of presentation, as well as contextual effects that arise from the order of items. In particular, since discrimination is hindered when a second token is presented rapidly (i.e., $<250 \mathrm{msec}$ ) after an unambiguous token, populations with slow phonological processing (for a review, see Habib, 2000) may be more differentially vulnerable to this contextual effect. This vulnerability could manifest itself as a differential onset time, duration, and/or perhaps magnitude of the context effect. A test of this hypothesis is underway in adults with reading disabilities.

\section{REFERENCES}

Adlard, A., \& Hazan, V. (1998). Speech perception in children with specific reading difficulties (dyslexia). Quarterly Journal of Experimental Psychology: Human Experimental Psychology, 51A, 153-177. 
Allen, J. S., \& Miller, J. L. (2001). Contextual influences on the internal structure of phonetic categories: A distinction between lexical status and speaking rate. Perception \& Psychophysics, 63, 798-810.

Breier, J. I., Gray, L., Fletcher, J. M., Diehl, R. L., KlaAs, P., FoORMAN, B. R., \& Molis, M. R. (2001). Perception of voice and tone onset time continua in children with dyslexia with and without attention deficit/hyperactivity disorder. Journal of Experimental Child Psychology, 80, 245-270.

DieHL, R. L., Elman, J. L., \& McCusker, S. B. (1978). Contrast effects on stop consonant identification. Journal of Experimental Psychology: Human Perception \& Performance, 4, 599-609.

DiEHL, R. L., LANG, M., \& PARKER, E. M. (1980). A further parallel between selective adaptation and contrast. Journal of Experimental Psychology: Human Perception \& Performance, 6, 24-44.

EIMAS, P. D., \& CoRBIT, J. D. (1973). Selective adaptation of linguistic feature detectors. Cognitive Psychology, 4, 99-109.

Grossberg, S., \& Myers, C. W. (2000). The resonant dynamics of speech perception: Interword integration and duration-dependent backward effects. Psychological Review, 107, 735-767.

Навів, M. (2000). The neurological basis of developmental dyslexia: An overview and working hypothesis. Brain, 123, 2374-2399.

IVERSON, P., \& KUHL, P. K. (1996). Influences of phonetic identification and category goodness on American listeners' perception of $/ \mathrm{r} /$ and /1/. Journal of the Acoustical Society of America, 99, 1130-1140.

Iverson, P., \& KUHL, P. K. (2000). Perceptual magnet and phoneme boundary effects in speech perception: Do they arise from a common mechanism? Perception \& Psychophysics, 62, 874-886.

KuHL, P. K. (1991). Human adults and human infants show a "perceptual magnet effect" for the prototypes of speech categories, monkeys do not. Perception \& Psychophysics, 50, 93-107.

KunL, P. K. (1994). Learning and representation in speech and language. Current Opinion in Neurobiology, 4, 812-822.

Lotto, A. J., \& KLUENDER, K. R. (1998). General contrast effects in speech perception: Effect of preceding liquid on stop consonant identification. Perception \& Psychophysics, 60, 602-619.

LotTo, A. J., KLUENDER, K. R., \& Holt, L. L. (1998). Depolarizing the perceptual magnet effect. Journal of the Acoustical Society of America, 103, 3648-3655.

MACMILlan, N. A., Goldberg, R. F., \& Braida, L. D. (1988). Resolution for speech sounds: Basic sensitivity and context memory on vowel and consonant continua. Journal of the Acoustical Society of America, 84, 1262-1280.

Manis, F. R., McBride-Chang, C., Seidenberg, M. S., Keating, P., Doi, L. M., Munson, B., \& Petersen, A. (1997). Are speech perception deficits associated with developmental dyslexia? Journal of Experimental Child Psychology, 66, 211-235.

Masterson, J., Hazan, V., \& WiJayatilake, L. (1995). Phonemic processing problems in developmental phonological disorders. Cognitive Neuropsychology, 12, 233-259.

Mody, M., Studdert-KenNedy, M., \& Brady, S. (1997). Speech perception deficits in poor readers: Auditory processing or phonological coding? Journal of Experimental Child Psychology, 64, 199-231.

Samuel, A. G. (1982). Phonetic prototypes. Perception \& Psychophysics, 31, 307-314.

SCHOUTEN, M. E., \& VAN HESSEN, A. J. (1992). Modeling phoneme perception. I: Categorical perception. Journal of the Acoustical Society of America, 92, 1841-1855.

TALlal, P., \& PIERCY, M. (1974). Developmental aphasia: Rate of auditory processing and selective impairment of consonant perception. Neuropsychologia, 12, 83-93.

Thibodeau, L. M., \& Sussman, H. M. (1979). Performance on a test of categorical perception of speech in normal and communication disordered children. Journal of Phonetics, 7, 375-391.

Werker, J. F., \& TEEs, R. C. (1987). Speech perception in severely disabled and average reading children. Canadian Journal of Psychology, 41, 48-61.

\section{APPENDIX}

The synthesized /ba/-/pa/ stimuli were adapted from a continuum developed by Tom Carrell (available at http://hush.unl.edu/ LabResources.html). The 11 tokens in the continuum spanned a VOT range from 0 to $50 \mathrm{msec}$ incrementing in approximately 5 -msec steps where VOT was measured from the end of the 5 -msec burst to the beginning of a full pitch pulse of the following vowel. The synthesized speech represented a male voice, and the first three formant frequencies were 660,1100, and $2500 \mathrm{~Hz}$ in the steady state of the vowel with a transition of approximately $30 \mathrm{msec}$ and an overall duration of $200 \mathrm{msec}$.

(Manuscript received May 8, 2003;

revision accepted for publication June 15, 2004.) 